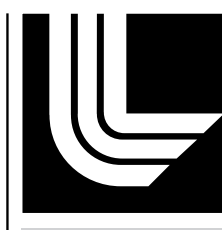

LAWRENCE LIVERMORE NATIONAL LABORATORY
Shear Localization and Failure in Shocked Metals Final Report

G.H. Campbell, F.X. Garaizar, M. Kumar, D.P. Trebotich, J.S. Stolken, and J.M. McNaney

18 February 2004

LDRD ER project 01-ERD-026 
This document was prepared as an account of work sponsored by an agency of the United States Government. Neither the United States Government nor the University of California nor any of their employees, makes any warranty, express or implied, or assumes any legal liability or responsibility for the accuracy, completeness, or usefulness of any information, apparatus, product, or process disclosed, or represents that its use would not infringe privately owned rights. Reference herein to any specific commercial product, process, or service by trade name, trademark, manufacturer, or otherwise, does not necessarily constitute or imply its endorsement, recommendation, or favoring by the United States Government or the University of California. The views and opinions of authors expressed herein do not necessarily state or reflect those of the United States Government or the University of California, and shall not be used for advertising or product endorsement purposes. 


\title{
Shear Localization and Failure in Shocked Metals Final Report
}

\author{
LDRD ER project 01-ERD-026
}

\author{
(P.I.), F. Xabier Garaizar, Mukul Kumar, David P. Trebotich, James S. \\ Stolken, and James M. McNaney
}

\section{Introduction}

The goal of the project was to understand the effect of shocks on the subsequent mechanical response of metals. The framework revolves around the sequence and timing of events during shock loading. A shock will transmit through a solid at speed of several $\mathrm{mm}$ per $\mu \mathrm{s}$. The result of the shock passage is a step change in the velocity of the material. This subsequent velocity will cause deformation in the material that could extend in time to several 10s or 100s of $\mu \mathrm{s}$ after the passage of the shock. How the material responds in this timeframe after shock passage is intimately related to its mechanical properties. The mechanical properties of interest are the stress-strain response, the susceptibility to localization, and the failure process. In short, the shock passes through a material first before it has time to move, however it does send the material into motion that causes mechanical deformation and usually some sort of failure.

The passage of a shock through a metal causes a dramatic change in the microstructure of that metal. The state of high uniaxial compression in a shock front causes all possible dislocation sources to become active and pumps the microstructure full of dislocations. The dislocation content of a microstructure is the single most important factor in determining the plastic response of the metal. The motion of dislocations through a crystal is the mechanism by which a metal plastically deforms. Due to dislocationdislocation interactions, the motion of a given dislocation through a crystal becomes progressively more difficult as the concentration of dislocations increases. This phenomenon is the mechanism by which a metal work hardens; as deformation progresses and dislocation sources raise the dislocation concentration it becomes increasingly difficult for dislocations to move. We would expect a similar process to occur for shocked material.

The ability to simulate large plastic deformation of materials is often limited in Legrangian finite element codes. Likewise, the ability to simulate material failure is limited on many standard types of simulation code. Therefore we decided to explore the possibility of building a simulation tool that would not be limited by these considerations. A basic framework of a Eulerian simulation code was developed and successfully tested. We feel that this code could form the basis upon which to build capabilities for 
performing materials dynamics simulations that include physical models of materials microstructure and more realistic models of materials failure.

The aims of the experimental aspects of this project were to gain an understanding of the physics involved with the failure process such that predictive, physics-based failure and fracture models can be developed in the future by incorporating microstructure and failure processes into a simulation code.

\section{Code development}

The modeling and simulation effort centered on building a simulation capability suited to the unique challenges posed by fracture and failure. The large strains associated with ductile failure dictates the use of an Eulerian code. The approach incorporates explicitly conservative schemes in the quantities of energy, density, and momentum and so should predict wave structures accurately. The code is inherently three-dimensional, parallel in implementation, and incorporate adaptive mesh refinement. Building the code with the SAMRAI toolbox developed in CASC enables these capabilities. A working version of the code now exists and simulations shown in Figures 1 and 2 are example of the capability.

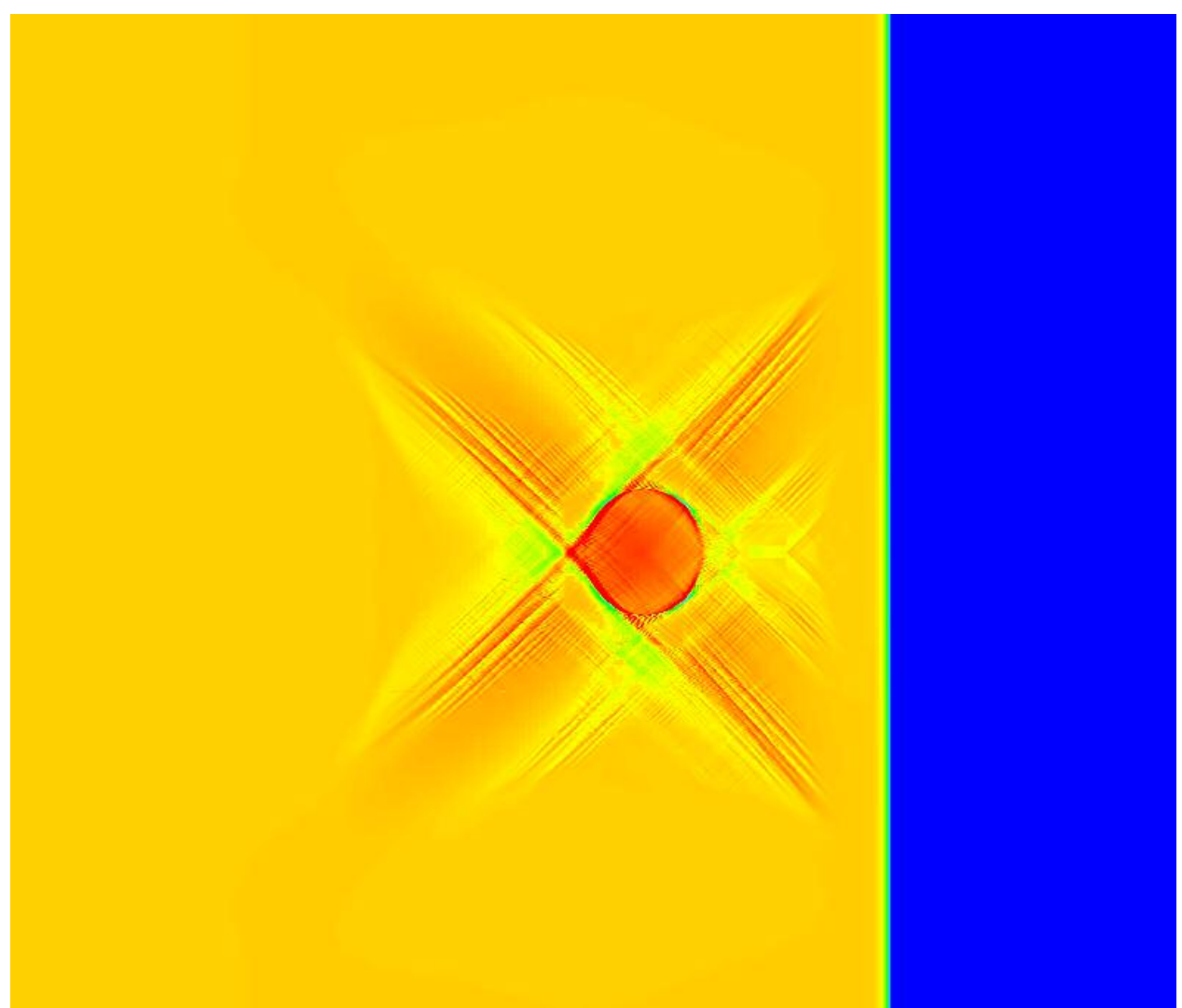

Figure 1 - Simulation of a shock interacting with a soft inclusion in $C u$. The shock has transited from left to right, passing through the inclusion, and is located at the transition from yellow to blue. The color scheme indicates levels of plastic strain. 


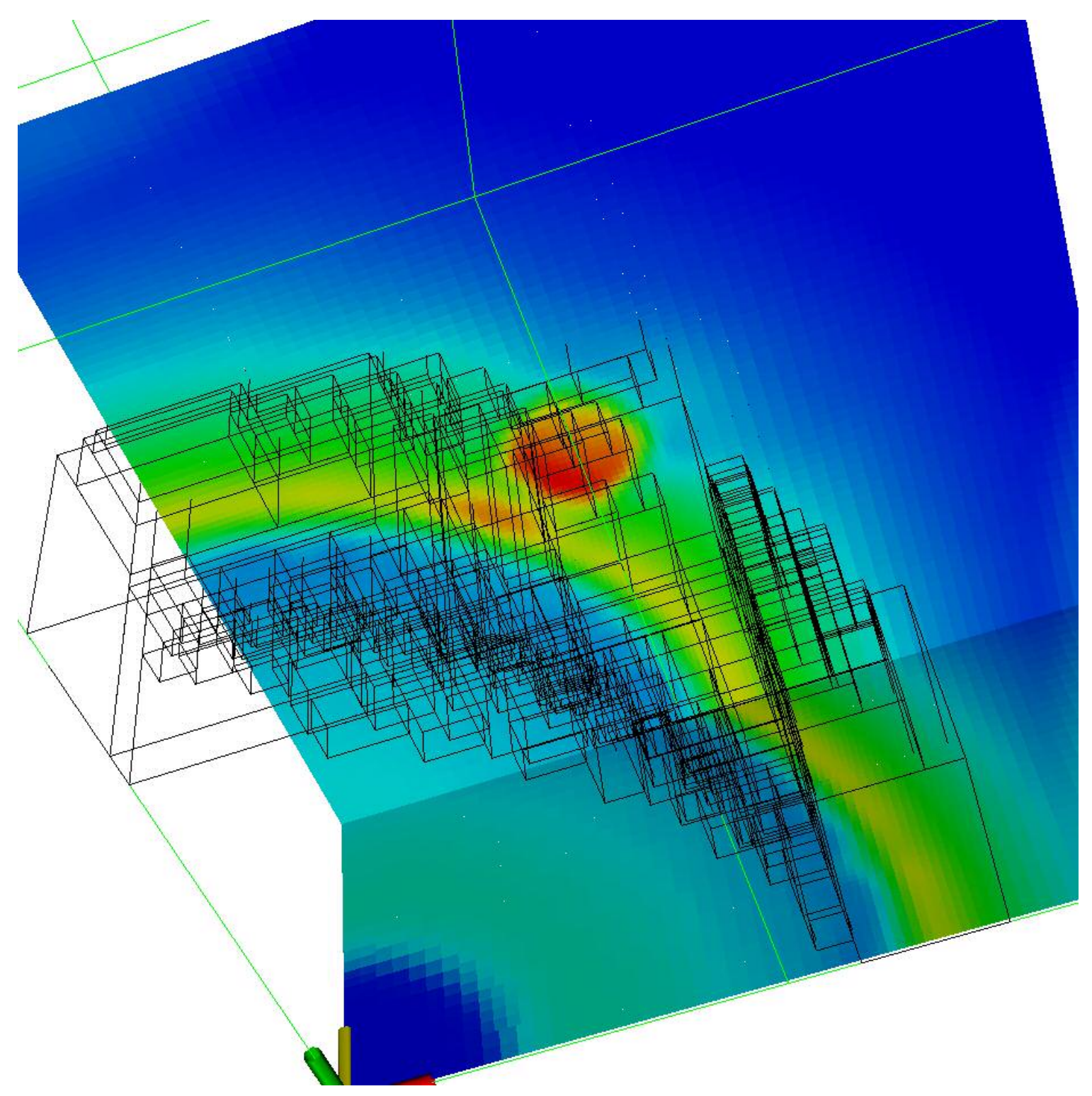

Figure 2 - An example of a three dimensional simulation, this time of a spherical shock front propagating outwards and interacting with an inclusion. The color scheme again indicates levels of plastic strain. The mesh has been superimposed on the image to show the adaptive mesh capabilities.

The advanced formulation of the material EOS used in the code is fully described in the accompanying document, "Implementation of an EOS and Strength Model for Metals in Conservative Form" (UCRL-TR-202310) by David Trebotich and James Stolken. This state of progress in the code development was as it stood at the end of the second year of the project. This component of the project was not funded in the third and final year. 


\section{Experimental Results}

A metal loaded by $\mathrm{HE}$ will first experience a strong shock from the HE detonation. This shock dramatically alters certain important aspects of the metal microstructure. During the shock loading, dislocation sources are activated and, due to the limitation of dislocation velocity to the speed of sound, the duration of the shock pulse plays an important role in determining the subsequent dislocation concentration. Also, at the shock pressures associated with HE, many materials will undergo twinning or other shear deformation modes. However, some aspects of the original microstructure will remain unaltered as a result of the shock, these include grain size and shape, grain boundary character, texture, and the presence of impurities or second phase particles. Nevertheless, it is the interaction of the dislocation content and spatial structure with these other aspects of the microstructure that determines the mechanical response of the metal and its subsequent failure and fracture behavior. Understanding these relationships is essential to developing a mechanism-based framework of material failure that includes the important physics of metal mechanical response.

Laser shock processing (LSP) was used to produce the relevant microstructures. We have shock processed the three materials of interest $(\mathrm{Cu}, \mathrm{Ta}$, and $\mathrm{Ta}-10 \% \mathrm{~W})$ at differing laser fluences and characterized the induced microstructural changes in order to identify the appropriate processing conditions. We have identified the spall threshold for each materials so that it can be avoided. Typically, we induce the largest shock pressure possible while avoiding spall. These shock pressures typically are less than $10 \mathrm{GPa}$. We have characterized the microstructure of the shock-recovered materials by transmission electron microscopy (TEM). The primary change in microstructure is in the dislocation content, making TEM the best-suited characterization technique. An example of the change in microstructure is shown in Figure 3. Based on these microstructural investigations, we have chosen the laser fluences of $20 \mathrm{~J} / \mathrm{cm}_{2}$ for $\mathrm{Cu}, 40 \mathrm{~J} / \mathrm{cm}_{2}$ for Ta, and $150 \mathrm{~J} / \mathrm{cm}_{2}$ for $\mathrm{Ta}-10 \% \mathrm{~W}$. Additionally, two shocked states have been considered: one
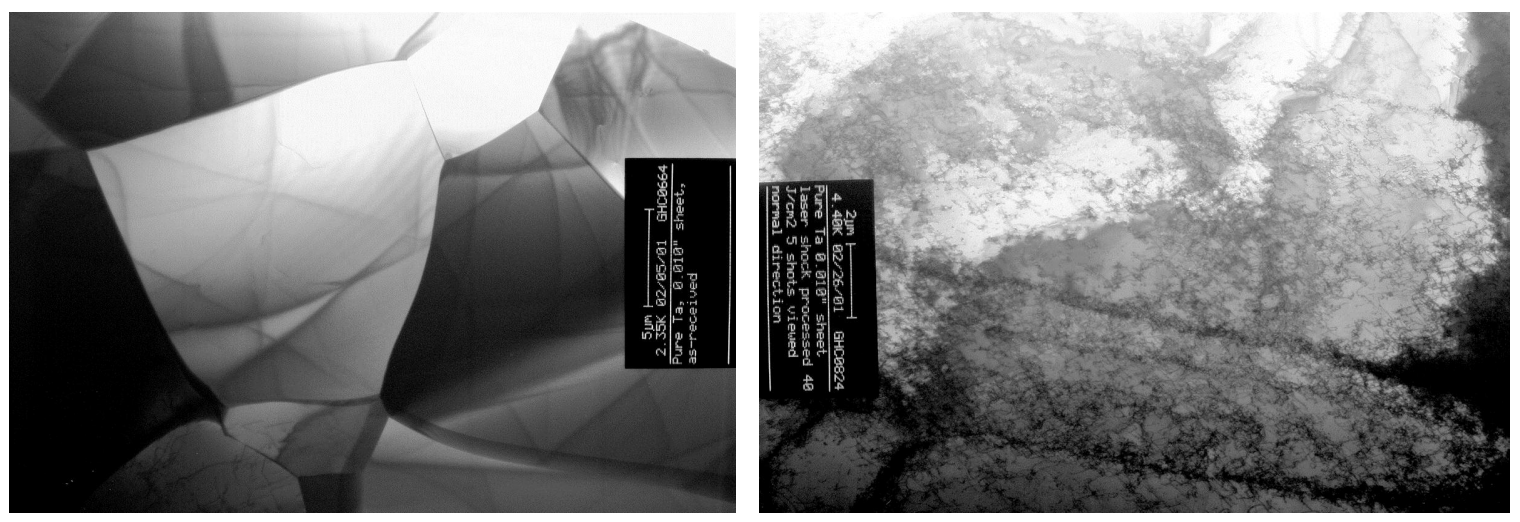

Figure 3-Transmission electron micrographs comparing the microstructure of Ta before and after laser shock processing. On the left is before and on the right is after. The grains are seen to be nearly dislocation free on the left side. However the image on the right show a very high concentration of dislocations, seen as short squiggly dark lines in the image. 
pass with the laser over both the front and back sides of the specimen and 5 passes. The mechanical test specimens are $1 \mathrm{~mm}$ thick and the microstructural modifications created by the shock treatments appear to be homogeneously distributed through the thickness. The 5 passes of the shock treatment act to increase the dislocation content of the specimens and brings their microstructural state into close similarity to what has been observed in HE loaded material.

We performed mechanical tests to measure the constitutive response of the three materials in both the unshocked and two shock recovered conditions. The mechanical tests have been carried out at strain rates of $10^{-4} \mathrm{~s}^{-1}, 10^{0} \mathrm{~s}^{-1}$, and $7 \times 10^{3} \mathrm{~s}^{-1}$. An example of the high rate data acquired for the mechanical tests of copper are shown in Figure 4. It is evident that the laser shock processing has a dramatic effect on the mechanical response of the metal. The $\mathrm{Cu}$ data shows an increase in yield stress with shock processing, a decrease in work hardening rate, and a decrease in ultimate strain to failure. These are the types of behaviors that would be expected to create instabilities in the mechanical response and lead to localization of strain. These behaviors are related in greater detail and for the other loading rates in the accompanying manuscript: "Effect of Laser Shock Processing on the Microstructure and Mechanical Properties of Pure Cu", by G.H. Campbell and M. Kumar (UCRL-JC-150085). Also related there is the characterization

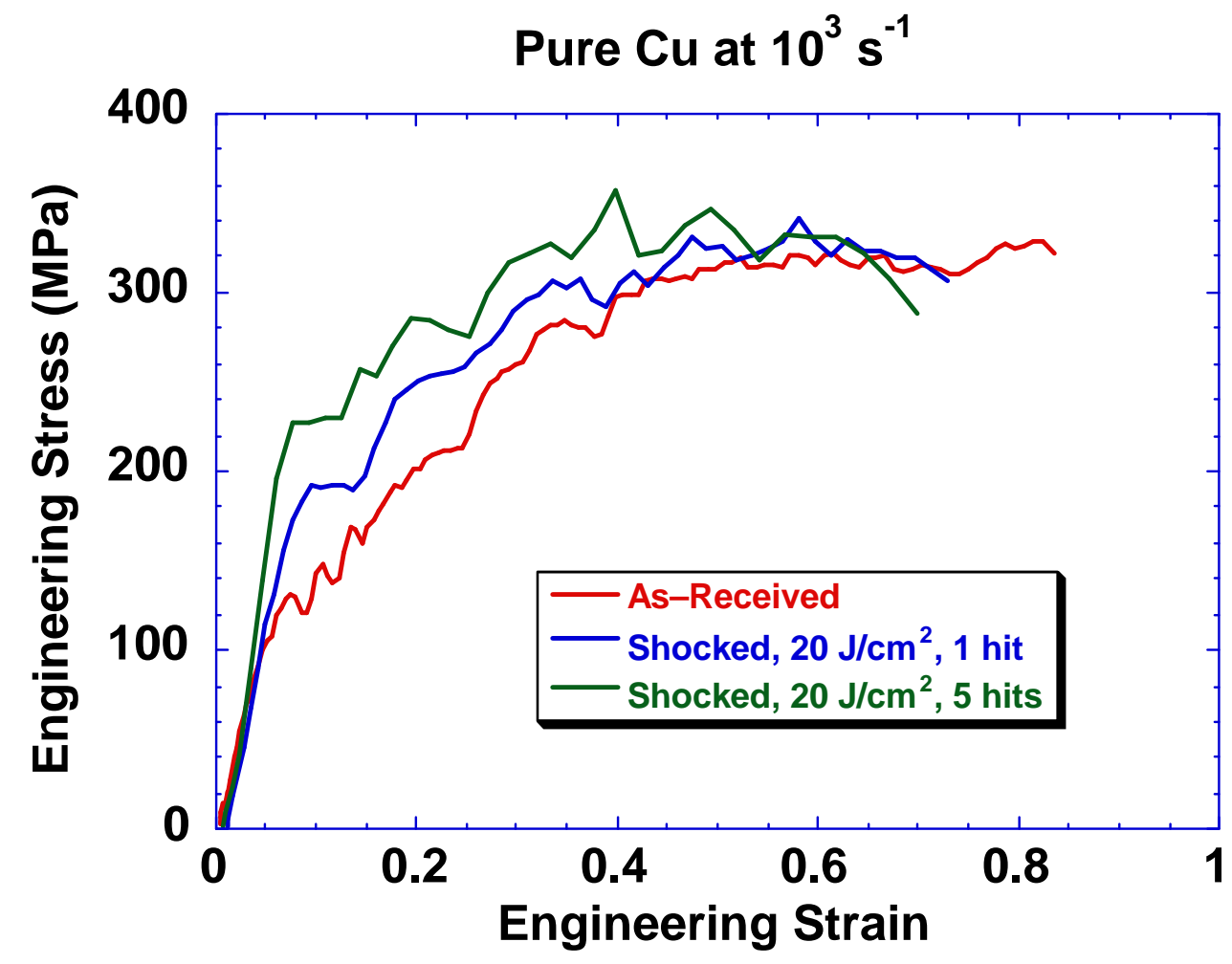

Figure 4 - The high strain rate mechanical properties of laser shocked $\mathrm{Cu}$ are revealed by tests on the split Hopkinson bar. The changes in the mechanical response are shown in the different curves. 
of the change in microstructure of the materials as a result of deformation during the mechanical tests. TEM specimens were taken from the gauge section of the test specimens. The evolution of the microstructure allows us to identify the physical mechanisms responsible for the changes in behavior revealed by the mechanical tests. For example, twin formation during straining.

\section{Material failure}

Ductile metals fail by void formation, growth, and linkage. This mechanism operates over a wide range of loading rates, from creep rupture $\left(d<10^{-7} \mathrm{~s}^{-1}\right)$ to spall $(\boldsymbol{d}>$ $\left.10^{6} \mathrm{~s}^{-1}\right)$. However, in this broad range of strain rates two distinct failure modes are observed. At the highest and lowest strain rates, voids form and grow simultaneously over large areas of the material. A distinct crack is absent. The voids link at a late stage in the failure process to form dividing surfaces. Here, damage mechanics are used to describe the failure process. Conversely, over a broad range of intermediate strain rates, materials fail by the formation of a crack and its extension. The crack is usually started at a pre-existing flaw in the microstructure, such as an inclusion or precipitate. At high deformation rates the crack may be preceded by the formation of an adiabatic shear band as a precursor structure. The presence of the crack modifies the stress field around it, causing a stress concentration at the crack tip. The concentrated stress causes deformation in the crack tip region that is generally referred to as the crack tip process zone. The stress concentration around cracks results in local load histories that are much different than the far field applied loads. The presence of cracks therefore requires an integrated fracture mechanics approach to understand the interplay between damage mechanisms and local loading which result in crack growth and ultimately material failure. A central component to the development of a failure framework upon which to build models is the identification and characterization of critical phenomena within the crack-tip process zone.

We have established a testing methodology to measure the fracture energy in plane stress for our materials. We are using a double edge notched tension geometry. We pre-crack the specimens by fatigue loading, which nucleates cracks symmetrically from the roots of both notches. Fatigue cracks are sharp at their tips due to the mechanism of formation and thus provide a valid initiation geometry for crack growth measures. Standard linear elastic fracture mechanics testing generally requires the use of samples which are large compared to the size of the fracture process zone (e.g., plastic zone). The thickness of LSP material necessitates the use of elasto-plastic fracture mechanics. Elasto-plastic fracture mechanics considers a number of complicating factors including general non-linear material behavior and crack blunting prior to initiation. This approach to fracture measurement involves quantifying the various stages of the ductile fracture process and relating them to a scaling parameter for the crack tip stress and strain fields, $J$, where $J$ is a path independent line integral. In practice, one uses standard fracture geometries and test procedures such as the double edge notch sample loaded in pure tension, used in this study. The results of the fracture tests, along with the tensile properties of the material and tabulated solutions from fully plastic finite element calculations, are used to determine the fracture energy, J. For a given material thickness, 
this fracture energy is applicable over a wide range of geometries where the deformation mode is homogeneous plasticity. The methodology and equipment have been developed for fracture testing of the LSP materials at low to moderate strain rates. Preliminary tests on brass and copper have been carried out to identify any unforeseen issues with the fatigue pre-cracking and fracture test methods. For example, tests on mill-stock copper sheet material yielded a fracture energy of $35 \mathrm{~kJ} / \mathrm{m}_{2}$, which compares favorably with literature values. Additional scoping work has been completed using lacquer coatings to assess plastic zone shape and growth during fracture testing.

\section{Conclusions}

Over the course of this project we have achieved the vast majority of its goals. We now understand the changes that are wrought on a metal's microstructure as a result of laser shock processing. We have identified several transitions in material response as a function of increasing shock pressure, from dislocation source activation, to dislocation banding, to twinning, and ultimately to spall failure. We have characterized these microstructures with several techniques, including transmission electron microscopy, scanning electron microscopy, electron backscatter diffraction mapping, optical microscopy, and electron diffraction. We have also measured how these shock induced microstructures impact the subsequent mechanical properties of the metals studied. We found substantial changes in the mechanical response and those changes are such that the material will become more susceptible to strain localization and premature failure. We have also developed the fracture mechanics methodology to measure the changes in fracture energy caused by these changes in microstructure. Additionally, we have developed the basic implementation of a simulation code that could in future incorporate the fundamental physics necessary to accurately simulate the types of behavior that we have observed in our experiments.

This work performed under the auspices of the U.S. Department of Energy by the University of California, Lawrence Livermore National Laboratory under contract No. W-7405-Eng-48. 